\title{
The accretion-diffusion scenario for metals in cool white dwarfs
}

\author{
D. Koester and D. Wilken
}

\author{
Institut für Theoretische Physik und Astrophysik, University of Kiel, 24098 Kiel, Germany \\ e-mail: koester@astrophysik.uni-kiel.de
}

Received 11 January 2006 / Accepted 31 January 2006

ABSTRACT

\begin{abstract}
We calculated diffusion timescales for $\mathrm{Ca}, \mathrm{Mg}, \mathrm{Fe}$ in hydrogen atmosphere white dwarfs with temperatures between 5000 and $25000 \mathrm{~K}$. With these timescales we determined accretion rates for a sample of $38 \mathrm{DAZ}$ white dwarfs from the recent studies of Zuckerman et al. (2003, ApJ, 596, 477) and Koester et al. (2005, A\&A, 432, 1025). Assuming that the accretion rates can be calculated with the Bondi-Hoyle formula for hydrodynamic accretion, we obtained estimates for the interstellar matter density around the accreting objects. These densities are in good agreement with new data about the warm, partially ionized phase of the ISM in the solar neighborhood.
\end{abstract}

Key words. stars: white dwarfs - stars: abundances

\section{Introduction}

Gravitational separation of elements in the strong gravitational field in the outer layers of white dwarfs has been known for a long time to be the primary physical process determining the atmospheric composition (Schatzman 1947). In the absence of competing processes (stellar wind, radiative levitation, convection), the heavy elements diffuse downward on a timescale that is always short compared to the evolutionary timescale, leaving the lightest element that is present floating on top. In most cases this is hydrogen, leading to the spectral type DA; if no hydrogen is present, the atmosphere is pure He (spectral types DB, DC).

However, there are some exceptions to this rule. In cooler white dwarfs below $25000 \mathrm{~K}$, which are the topic of this study, we sometimes find traces of $\mathrm{Ca}$ (through the strong CaII resonance lines), occasionally accompanied by $\mathrm{Mg}$ and Fe. For Herich atmospheres this has been known from the time of the first white dwarfs discovered, since the well known vMa 2 is a member of the class now called DZ ( $\mathrm{Z}$ always stands for the presence of metals, DZ means no other elements visible). The class of hydrogen-rich objects with metals (DAZ, with visible hydrogen and metal lines) has really only come into existence over the past 8 years with the detection of a few dozen members in large surveys at the Keck and VLT telescopes (Zuckerman \& Reid 1998; Zuckerman et al. 2003; Koester et al. 2005). The reason for this difference is purely observational bias; because of the much lower transparency of a $\mathrm{H}$ atmosphere at these temperatures, the CaII equivalent widths in the DAZs are about a factor 1000 smaller than in the DZs at the same abundance.

The diffusion timescales for the heavy elements to disappear from the atmosphere - or in case of an outer convection zone from the bottom of this zone - is always short compared to the cooling timescale. A possibly competing process, radiative levitation, becomes completely negligible for $\mathrm{Ca}$ below $T_{\text {eff }}=25000 \mathrm{~K}$ (Chayer et al. 1995). This implies that the observed metals cannot be primordial. They must have been supplied from the outside, and currently the most widely accepted mechanism for this is accretion of interstellar matter. There are still some problems with this scenario, the most serious being the absence of cool, dense clouds in the ISM of the solar neighborhood. Aannestad et al. (1993) tried to correlate the positions and motions of DZ (helium-rich) stars with conditions of the ISM, but with inconclusive results. Alternative explanations are therefore still being considered by several authors (see e.g. the discussion in Zuckerman et al. 2003).

Because the timescales for diffusion and thus metal visibility in the DZs typically are on the order of $10^{6} \mathrm{yr}$ (Paquette et al. 1986b; see also Sect. 4), the stars could have traveled a large distance (of order $50 \mathrm{pc}$ ) since the accretion ended, so that a lack of correlation is not too surprising. This situation has changed dramatically with the recent detection of a large number of DAZs. In these hydrogen-rich atmospheres the diffusion timescales are about 3-4 orders of magnitude shorter, practically meaning that we can only expect to observe metals if the accretion is still going on at the present time. The DAZs thus offer two advantages compared to the (helium-rich) DZs

- we know where the accretion occurs and can compare directly with the conditions of the ISM in that region;

- we argue that we very likely observe a steady state accretion/diffusion, meaning that we can determine the accretion rate responsible for the observed abundances.

There is thus a much more promising chance to learn something about the accretion process and/or the conditions in the ISM from an analysis of the observed metals in the DAZs.

\section{The accretion-diffusion scenario}

The idea that metals are accreted onto white dwarfs and then diffused downward out of sight has been developed by many authors. It was finally explored in great detail and transformed into a clear and testable theory in a series of three fundamental papers by the Montreal group (Dupuis et al. 1992, 1993a,b). The basic idea of the so-called "two-phase accretion model" is that the white dwarf travels for most of the time in the hot tenuous phase of the ISM with negligible accretion rates. After typically $5 \times 10^{7} \mathrm{yr}$, it enters a dense cloud, where the accretion rate is high and the metal abundance in the outer layers approaches an equilibrium value between accretion and diffusion. After about 
$10^{6} \mathrm{yr}$ traveling through the cloud, the star emerges and the metal abundance decreases exponentially with the diffusion timescale.

The observations available at that time - almost exclusively of DZs and DBZs - agreed quite well with the predictions of this model, although it obviously is an extreme oversimplification as emphasized by the authors. It was subsequently called into question with the findings that the required dense neutral clouds, which would be detectable through $\mathrm{Na}$ I absorption, are not found within the Local Bubble, where these white dwarfs are located (Sfeir et al. 1999; Welsh et al. 1998, 1999; Redfield \& Linsky 2002; Lehner et al. 2003). Nevertheless we will keep this model as our first hypothesis for this study since it is specific enough to make quantitative, testable predictions.

While dense, cool gas seems to be absent in the Local Bubble, the presence of warm, partially ionized gas has been clearly demonstrated by Redfield \& Linsky (2002, 2004a). The average number density of neutral hydrogen is around $n_{\mathrm{HI}}=$ $0.1 \mathrm{~cm}^{-3}$, but very little is known about the spatial distribution. It is also unclear whether accretion under these conditions would follow the Bondi-Hoyle rate (see Koester et al. 2005). However, following arguments explained below, and in order to have a different model for testing against the standard assumptions, we will define our alternative hypothesis:

- the H-rich white dwarfs observed with metals are currently accreting in a steady state, with accretion and diffusion proceeding at the same rate;

- there are no large-scale differences (like the two phases of the standard model). Many, and perhaps all, white dwarfs in the Local Bubble are accreting with a continuous distribution of accretion rates caused by fluctuations in ISM densities and differences in the stellar parameters. The ones we can identify as DAZs are the top of the distribution.

Obviously this and the Dupuis et al. hypothesis are simplifications at extreme ends of the possibilities and could be characterized as "two-phase" vs. "continuous" accretion. In the following sections we explore whether the observations can lead to a distinction between the alternatives.

\section{The observed sample}

The data come from two large surveys: a search for Ca in DAs using the Keck telescope and the ESO Supernova Ia Progenitor Survey (SPY). The instruments and reduction procedures are described in Zuckerman et al. (2003) and Koester et al. (2005). Atmospheric parameters $T_{\text {eff }}, \log g$, and Ca abundances are given in those papers and in Berger et al. (2005). Taking both sources together, we have a sample of 38 DAZs (excluding those marked as double degenerates or DA+DM pairs in Zuckerman et al. 2003) with observed photospheric CaII lines and about 450 DAs with upper limits for the $\mathrm{Ca}$ abundance.

Table 1 collects the data for the DAZs, while Fig. 1 graphically shows the distribution of $\mathrm{Ca}$ abundances with $T_{\text {eff }}$. The line corresponds to an equivalent width of the CaII K line of $15 \mathrm{~m} \AA$, which is the observational limit for our spectra with the highest signal-to-noise ratio. Because of the ionization of CaII to CaIII with increasing effective temperature, the lower limit for observable $\mathrm{Ca}$ increases until it becomes unobservable between 20000 and $25000 \mathrm{~K}$. For all objects with $\mathrm{Ca}$ lines in our sample, the photospheric nature (as opposed to interstellar absorption) was confirmed through a comparison of radial velocity determinations from the $\mathrm{Ca}$ and the hydrogen Balmer lines.

$\mathrm{Ca}$ is found at all temperatures filling the area of abundances between the lower observational limit and some upper limit
Table 1. Data for 38 objects with detected photospheric Ca. Atmospheric parameters $T_{\text {eff }}, \log g$, and logarithmic Ca/H ratio, $[\mathrm{Ca} / \mathrm{H}]$, are from Zuckerman et al. (2003), Koester et al. (2005), or Berger et al. (2005). Space velocities, $v$, relative to the sun are from Pauli (2003), Zuckerman et al. (2003). In eight cases where the space velocity is not known, we have replaced it with $\sqrt{3}$ times the radial velocity, taken from the cited reference. Column $d$ is the distance in pc, taken from Pauli (2003), McCook \& Sion (1999).

\begin{tabular}{lrrrrr}
\hline \hline Object & $\begin{array}{r}T_{\text {eff }} \\
{[\mathrm{K}]}\end{array}$ & $\log g$ & {$[\mathrm{Ca} / \mathrm{H}]$} & $\begin{array}{r}v \\
{\left[\mathrm{~km} \mathrm{~s}^{-1}\right]}\end{array}$ & $\begin{array}{r}d \\
{[\mathrm{pc}]}\end{array}$ \\
\hline WD0032-175 & 9235 & 8.0 & -10.5 & 74 & 32 \\
HS0047+1903 & 16600 & 7.8 & -6.1 & 5 & \\
HE0106-3253 & 15700 & 8.0 & -5.8 & 20 & 66 \\
WD0208+296 & 7201 & 7.9 & -8.8 & 89 & \\
WD0235+064 & 11420 & 7.9 & -9.2 & 11 & 34 \\
WD0243-026 & 6798 & 8.2 & -9.8 & 52 & 21 \\
WD0245+541 & 5190 & 8.2 & -11.7 & 38 & 10 \\
HS0307+0746 & 10200 & 8.1 & -7.1 & 38 & \\
WD0408-041 & 14400 & 7.8 & -6.6 & 40 & 74 \\
WD0543+579 & 8142 & 8.0 & -10.5 & 120 & 30 \\
WD0846+346 & 7373 & 8.0 & -9.4 & 20 & 27 \\
WD1015+161 & 19300 & 7.9 & -5.9 & 47 & 95 \\
WD1102-183 & 8026 & 8.0 & -10.3 & 45 & 40 \\
WD1116+026 & 12200 & 7.9 & -6.5 & 26 & 43 \\
WD1124-293 & 9700 & 8.1 & -8.2 & 65 & 34 \\
WD1150-153 & 12800 & 7.8 & -6.0 & 14 & 85 \\
WD1202-232 & 8800 & 8.2 & -9.8 & 37 & 10 \\
WD1204-136 & 11200 & 8.0 & -7.2 & 56 & 52 \\
WD1208+576 & 5830 & 7.9 & -10.8 & 65 & 20 \\
HE1225+0038 & 9400 & 8.1 & -9.7 & 38 & 29 \\
WD1257+278 & 8481 & 7.9 & -8.1 & 50 & 35 \\
HE1315-1105 & 9400 & 8.4 & -9.4 & 17 & 40 \\
WD1337+705 & 20435 & 7.9 & -6.7 & 53 & 33 \\
WD1344+106 & 6945 & 8.0 & -11.3 & 79 & 18 \\
WD1407+425 & 9856 & 8.0 & -9.8 & 21 & 26 \\
WD1455+298 & 7366 & 7.6 & -9.3 & 101 & 35 \\
WD1457-086 & 20400 & 8.0 & -6.1 & 24 & 117 \\
WD1614+160 & 17400 & 7.8 & -7.2 & 44 & 117 \\
WD1633+433 & 6569 & 8.1 & -8.6 & 30 & 15 \\
WD1821-131 & 7029 & 8.4 & -10.9 & 89 & 20 \\
WD1826-045 & 9200 & 8.1 & -8.6 & 26 & 25 \\
WD1858+393 & 9470 & 8.0 & -7.8 & 3 & 35 \\
WD2115-560 & 9700 & 8.1 & -7.6 & 54 & 22 \\
HS2132+0941 & 13200 & 7.7 & -7.1 & 37 & 79 \\
WD2149+021 & 17300 & 7.9 & -7.7 & 4 & 25 \\
HE2221-1630 & 10100 & 8.2 & -7.2 & 25 & 52 \\
HS2229+2335 & 18600 & 7.9 & -5.9 & 35 & 91 \\
WD2326+049 & 12100 & 7.9 & -6.4 & 59 & 19 \\
\hline
\end{tabular}

$[\mathrm{Ca} / \mathrm{H}] \approx-5.7([\mathrm{Ca} / \mathrm{H}]$ is the logarithmic number ratio of the elements). This upper limit decreases towards lower $T_{\text {eff }}$, which will be discussed in a later section.

\section{Diffusion timescales}

When trying to interpret the observational results in terms of an accretion-diffusion scenario, the most important theoretical ingredient is the diffusion timescale. In the absence of an outer supply, heavy elements disappear exponentially out of the reservoir (either the atmosphere or the outer convection zone, which is assumed homogeneously mixed) on a timescale given by (see e.g. Dupuis et al. 1992)

$\tau_{\mathrm{D}}=\frac{M_{\mathrm{WD}} q}{4 \pi r_{\mathrm{b}}^{2} \rho w}$ 


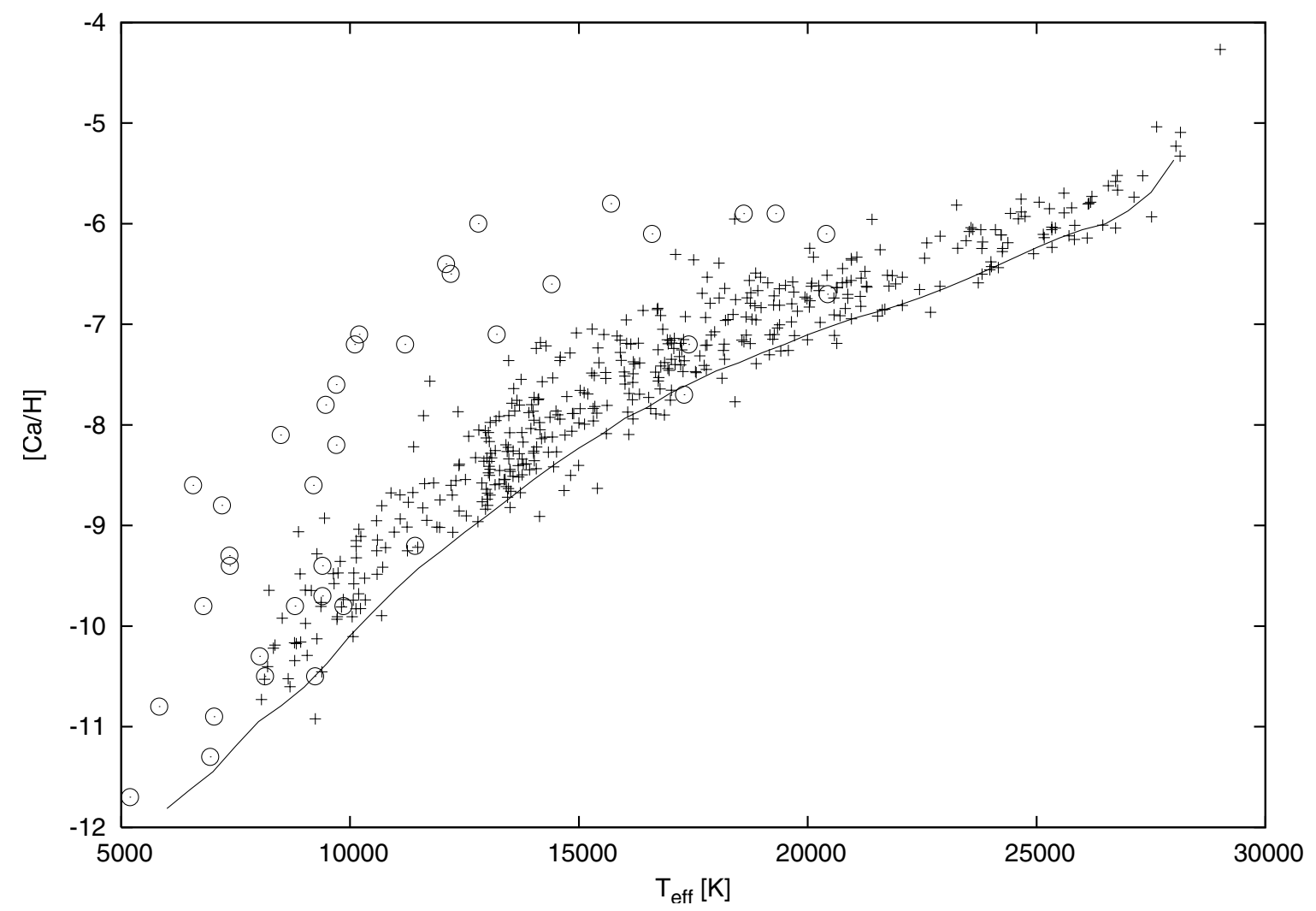

Fig. 1. Logarithmic $\mathrm{Ca}$ abundances (circles) and upper limits (crosses) for the combined sample (see text). The continuous line indicates a constant equivalent width of $E W=15 \mathrm{~m} \AA$, taken as observational limit for most objects.

where $M_{\mathrm{WD}}$ is the mass of the white dwarf, $q$ the mass fraction of the reservoir, $r_{\mathrm{b}}$ the radius at the bottom of the reservoir (always close to the white dwarf radius), $\rho$ the mass density, and $w$ the relative diffusion velocity between the heavy trace element and the main constituent, which is hydrogen in our case. Paquette et al. (1986b) discuss the influence of the parameters $q, \rho, w$ on the timescales, in particular with regard to the large differences between $\mathrm{He}$ and $\mathrm{H}$ envelopes. Basically the reason is the following: the convection zones $(q)$ are much thicker in He envelopes at the same mass and effective temperature. The density at the bottom increases, but not as fast as $q$, and in addition the velocity $w$ is smaller in denser environments. The combination of these factors adds up to give timescales for He several orders of magnitudes larger than for $\mathrm{H}$ at the same $T_{\text {eff }}$.

The Dupuis et al. papers only give timescales in helium atmospheres in a tabular form, and only a few calculations for hydrogen envelopes below $10000 \mathrm{~K}$, due to the lack of observational data at that time. Although Paquette et al. (1986b) give data for hydrogen envelopes up to $15000 \mathrm{~K}$, we nevertheless decided to repeat such calculations in order to obtain a homogeneous set of data covering the range of observations and incorporating the improved understanding of the mixing-length parameters in DA white dwarfs.

For these calculations we used our stellar atmosphere and stellar structure codes, which use up-to-date input physics for opacities and non-ideal gas effects in the equation of state. The most relevant fact for this study is the use of the mixing-length version ML2 instead of ML3, and a mixing length of 0.6 pressure scale heights. Various mixing-length approximations used in white dwarf modeling differ in the choice of three numerical constants of the model (see Fontaine et al. 1981; Tassoul et al. 1990, for a definition of the nomenclature). In short, the ML3 version is a more efficient convection with higher energy flux at the same temperature gradient. Necessary stellar parameters for the diffusion time calculations, e.g. the physical conditions at the bottom of the reservoir of heavy elements to be depleted, were obtained from these models. Convection zones start very shallow in DAs above $T_{\text {eff }} \approx 15000 \mathrm{~K}$, and the reservoir is the atmosphere. We define, somewhat arbitrarily, the limit at an optical depth of $\tau_{\text {Ross }}=5$. When a $0.6 M_{\odot}$ white dwarf cools down below $13000 \mathrm{~K}$, the bottom becomes deeper than this, and the reservoir is defined by the bottom of the convection zone. For a more (less) massive white dwarf, the transition occurs at a slightly higher (lower) $T_{\text {eff }}$.

Collision integrals for the diffusion velocities were obtained from the fits in Paquette et al. (1986a) and the calculations followed the prescriptions in Paquette et al. (1986b) very closely. One major exception was that the detailed distribution over ionization states was obtained from the models and average diffusion velocities determined instead of just using the dominant ions as in the latter work. In view of significant uncertainties in non-ideal gas effects (e.g. pressure ionization) or convection theory, we do not believe that the result will be more accurate, but this method avoids discontinuities in timescale changes with temperature of the models. Table 2 gives the resulting timescales for $\mathrm{Ca}, \mathrm{Mg}$, and $\mathrm{Fe}$ in hydrogen, while Fig. 2 shows the $\mathrm{Ca}$ data in graphic form. In general these results are very similar to those obtained by Paquette et al. (1986b) and earlier work (Muchmore $1979,1984)$. The main difference is that the steep increase in diffusion timescales towards cooler temperatures occurs at slightly lower $T_{\text {eff }}$ because of different formulations of the mixing-length theory used, e.g. ML3 in the case of Paquette et al. (1986b).

The first conclusion from these data is a confirmation of earlier results: the diffusion timescales are extremely short 
Table 2. Logarithm of the diffusion timescales (in years) for $\mathrm{Ca}, \mathrm{Mg}$, and $\mathrm{Fe}$ in hydrogen-rich white dwarfs as a function of effective temperature and surface gravity.

\begin{tabular}{|c|c|c|c|c|c|c|c|c|}
\hline \multirow[b]{2}{*}{$T_{\mathrm{eff}}[\mathrm{K}]$} & & \multicolumn{7}{|c|}{$\overline{\log g}$} \\
\hline & & 7.5 & 7.75 & 8.0 & 8.25 & 8.5 & 8.75 & 9.0 \\
\hline & $\mathrm{Ca}$ & 6.1353 & 5.7436 & 5.3045 & 4.7835 & 4.1353 & 3.4683 & 2.5939 \\
\hline \multirow[t]{3}{*}{5000} & $\mathrm{Mg}$ & 6.1629 & 5.7188 & 5.2500 & 4.8033 & 4.2051 & 3.5215 & 2.6560 \\
\hline & $\mathrm{Fe}$ & 6.1406 & 5.7159 & 5.2216 & 4.6712 & 3.9877 & 3.3064 & 2.4714 \\
\hline & $\mathrm{Ca}$ & 5.1778 & 4.6687 & 4.2179 & 3.8380 & 3.4399 & 3.0440 & 2.5471 \\
\hline \multirow[t]{3}{*}{6000} & $\mathrm{Mg}$ & 5.2526 & 4.7300 & 4.2702 & 3.8767 & 3.4632 & 3.0424 & 2.5036 \\
\hline & $\mathrm{Fe}$ & 5.1932 & 4.6841 & 4.2439 & 3.8780 & 3.4981 & 3.1134 & 2.5948 \\
\hline & $\mathrm{Ca}$ & 4.6835 & 4.1794 & 3.6554 & 3.1968 & 2.7003 & 2.2280 & 1.7375 \\
\hline \multirow[t]{3}{*}{7000} & $\mathrm{Mg}$ & 4.7280 & 4.2117 & 3.6735 & 3.2086 & 2.6994 & 2.2151 & 1.7150 \\
\hline & $\mathrm{Fe}$ & 4.6304 & 4.1124 & 3.5628 & 3.0924 & 2.5650 & 2.0642 & 1.5538 \\
\hline & $\mathrm{Ca}$ & 4.2452 & 3.7668 & 3.2781 & 2.8298 & 2.3413 & 1.8960 & 1.3673 \\
\hline \multirow[t]{3}{*}{8000} & $\mathrm{Mg}$ & 4.2724 & 3.7838 & 3.2839 & 2.8307 & 2.3366 & 1.8874 & 1.3521 \\
\hline & $\mathrm{Fe}$ & 4.1262 & 3.6333 & 3.1189 & 2.6570 & 2.1427 & 1.6812 & 1.1178 \\
\hline & $\mathrm{Ca}$ & 3.6618 & 3.2656 & 2.8340 & 2.4193 & 1.9883 & 1.5547 & 1.0580 \\
\hline \multirow[t]{3}{*}{9000} & $\mathrm{Mg}$ & 3.6729 & 3.2722 & 2.8328 & 2.4146 & 1.9811 & 1.5451 & 1.0462 \\
\hline & $\mathrm{Fe}$ & 3.4729 & 3.0716 & 2.6270 & 2.2022 & 1.7571 & 1.3075 & 0.7892 \\
\hline & $\mathrm{Ca}$ & 2.1235 & 2.1596 & 2.1125 & 1.8695 & 1.5642 & 1.1934 & 0.7282 \\
\hline \multirow[t]{3}{*}{10000} & $\mathrm{Mg}$ & 2.0589 & 2.1135 & 2.0940 & 1.8610 & 1.5580 & 1.1841 & 0.7134 \\
\hline & $\mathrm{Fe}$ & 1.9373 & 1.9567 & 1.9125 & 1.6605 & 1.3344 & 0.9397 & 0.4499 \\
\hline & $\mathrm{Ca}$ & 0.7272 & 1.0429 & 1.3574 & 1.3264 & 1.1629 & 0.9366 & 0.5481 \\
\hline \multirow[t]{3}{*}{10500} & $\mathrm{Mg}$ & 0.7107 & 0.9840 & 1.2991 & 1.2851 & 1.1402 & 0.9232 & 0.5323 \\
\hline & $\mathrm{Fe}$ & 0.6606 & 0.9191 & 1.1606 & 1.1139 & 0.9485 & 0.7065 & 0.2875 \\
\hline & $\mathrm{Ca}$ & -0.6754 & -0.2462 & 0.2184 & 0.4745 & 0.5944 & 0.5090 & 0.2723 \\
\hline \multirow[t]{3}{*}{11000} & $\mathrm{Mg}$ & -0.4665 & -0.1343 & 0.1712 & 0.4423 & 0.5441 & 0.4723 & 0.2487 \\
\hline & $\mathrm{Fe}$ & -0.8266 & -0.2525 & 0.1212 & 0.3335 & 0.3868 & 0.2845 & 0.0385 \\
\hline & $\mathrm{Ca}$ & -1.0396 & -1.2166 & -1.0994 & -0.5974 & -0.2729 & -0.1556 & -0.1807 \\
\hline \multirow[t]{3}{*}{11500} & $\mathrm{Mg}$ & -0.8548 & -1.0199 & -0.8862 & -0.5751 & -0.3171 & -0.1855 & -0.2240 \\
\hline & $\mathrm{Fe}$ & -1.2632 & -1.3974 & -1.1010 & -0.6656 & -0.4005 & -0.3263 & -0.4012 \\
\hline & $\mathrm{Ca}$ & -1.1486 & -1.4876 & -1.7497 & -1.6973 & -1.2673 & -0.9217 & -0.8417 \\
\hline \multirow[t]{3}{*}{12000} & $\mathrm{Mg}$ & -0.9675 & -1.3054 & -1.5580 & -1.4801 & -1.1788 & -0.9544 & -0.8612 \\
\hline & $\mathrm{Fe}$ & -1.3827 & -1.7171 & -1.9453 & -1.7734 & -1.3084 & -1.0355 & -0.9847 \\
\hline & $\mathrm{Ca}$ & -1.1597 & -1.5499 & -1.9446 & -2.2830 & -2.5642 & -2.8837 & -2.7075 \\
\hline \multirow{3}{*}{13000} & $\mathrm{Mg}$ & -0.9673 & -1.3627 & -1.7644 & -2.0971 & -2.3796 & -2.6888 & -2.5069 \\
\hline & $\mathrm{Fe}$ & -1.3675 & -1.7691 & -2.1787 & -2.5123 & -2.7764 & -3.0656 & -2.7119 \\
\hline & $\mathrm{Ca}$ & -1.1156 & -1.5065 & -1.8966 & -2.2858 & -2.6730 & -3.0616 & -3.3721 \\
\hline \multirow[t]{3}{*}{14000} & $\mathrm{Mg}$ & -0.9063 & -1.3008 & -1.6961 & -2.0920 & -2.4880 & -2.8880 & -3.1820 \\
\hline & $\mathrm{Fe}$ & -1.2870 & -1.6861 & -2.0857 & -2.4867 & -2.8887 & -3.2975 & -3.5648 \\
\hline & $\mathrm{Ca}$ & -1.0700 & -1.4602 & -1.8491 & -2.2354 & -2.6268 & -3.0241 & -3.4227 \\
\hline \multirow[t]{3}{*}{15000} & $\mathrm{Mg}$ & -0.8583 & -1.2519 & -1.6451 & -2.0368 & -2.4286 & -2.8253 & -3.2286 \\
\hline & $\mathrm{Fe}$ & -1.2172 & -1.6147 & -2.0126 & -2.4102 & -2.8097 & -3.2097 & -3.6109 \\
\hline & $\mathrm{Ca}$ & -1.0351 & -1.4223 & -1.8116 & -2.2034 & -2.5981 & -2.9955 & -3.3929 \\
\hline 16000 & $\mathrm{Mg}$ & -0.8205 & -1.2101 & -1.6031 & -1.9952 & -2.3871 & -2.7870 & -3.1872 \\
\hline & $\mathrm{Fe}$ & -1.1619 & -1.5572 & -1.9569 & -2.3555 & -2.7510 & -3.1483 & -3.5439 \\
\hline & $\mathrm{Ca}$ & -1.0070 & -1.3937 & -1.7838 & -2.1772 & -2.5744 & -2.9721 & -3.3693 \\
\hline 17000 & $\mathrm{Mg}$ & -0.7924 & -1.1822 & -1.5741 & -1.9612 & -2.3587 & -2.7580 & -3.1572 \\
\hline & $\mathrm{Fe}$ & -1.1056 & -1.5030 & -1.9037 & -2.2994 & -2.6988 & -3.0968 & -3.4931 \\
\hline & $\mathrm{Ca}$ & -0.9846 & -1.3722 & -1.7646 & -2.1596 & -2.5554 & -2.9530 & -3.3503 \\
\hline 18000 & $\mathrm{Mg}$ & -0.7697 & -1.1600 & -1.5492 & -1.9401 & -2.3369 & -2.7358 & -3.1344 \\
\hline & $\mathrm{Fe}$ & -1.0435 & -1.4465 & -1.8485 & -2.2492 & -2.6490 & -3.0523 & -3.4543 \\
\hline & $\mathrm{Ca}$ & -0.9681 & -1.3565 & -1.7488 & -2.1438 & -2.5397 & -2.9373 & -3.3346 \\
\hline 19000 & $\mathrm{Mg}$ & -0.7512 & -1.1406 & -1.5288 & -1.9234 & -2.3199 & -2.7184 & -3.1167 \\
\hline & $\mathrm{Fe}$ & -0.9809 & -1.3866 & -1.7916 & -2.1955 & -2.6010 & -3.0076 & -3.4125 \\
\hline & $\mathrm{Ca}$ & -0.9545 & -1.3433 & -1.7352 & -2.1304 & -2.5263 & -2.9239 & -3.3213 \\
\hline 20000 & $\mathrm{Mg}$ & -0.7364 & -1.1252 & -1.5148 & -1.9102 & -2.3065 & -2.7047 & -3.1027 \\
\hline & $\mathrm{Fe}$ & -0.9241 & -1.3308 & -1.7373 & -2.1447 & -2.5525 & -2.9617 & -3.3692 \\
\hline & $\mathrm{Ca}$ & -0.8771 & -1.2732 & -1.6684 & -2.0677 & -2.4669 & -2.8662 & -3.2657 \\
\hline 24000 & $\mathrm{Mg}$ & -0.7030 & -1.0903 & -1.4830 & -1.8772 & -2.2719 & -2.6675 & -3.0640 \\
\hline & $\mathrm{Fe}$ & -0.7958 & -1.1973 & -1.6004 & -2.0050 & -2.4111 & -2.8187 & -3.2277 \\
\hline
\end{tabular}

compared to evolutionary timescales, and also much shorter than diffusion timescales in He-rich envelopes. Over most of the observed range, they are much shorter than $1000 \mathrm{yr}$, going down to days at the high-temperature end; and only for the very coolest objects do the timescales approach 100000 yr. The steep increase in the timescales between 12500 and $10000 \mathrm{~K}$ is directly connected to the very strong increase in the depth of the convection zone, when the white dwarf cools through this temperature region. If the distribution in Fig. 1 is due to objects $a f$ ter the end of their accretion episode (note that an exponential 


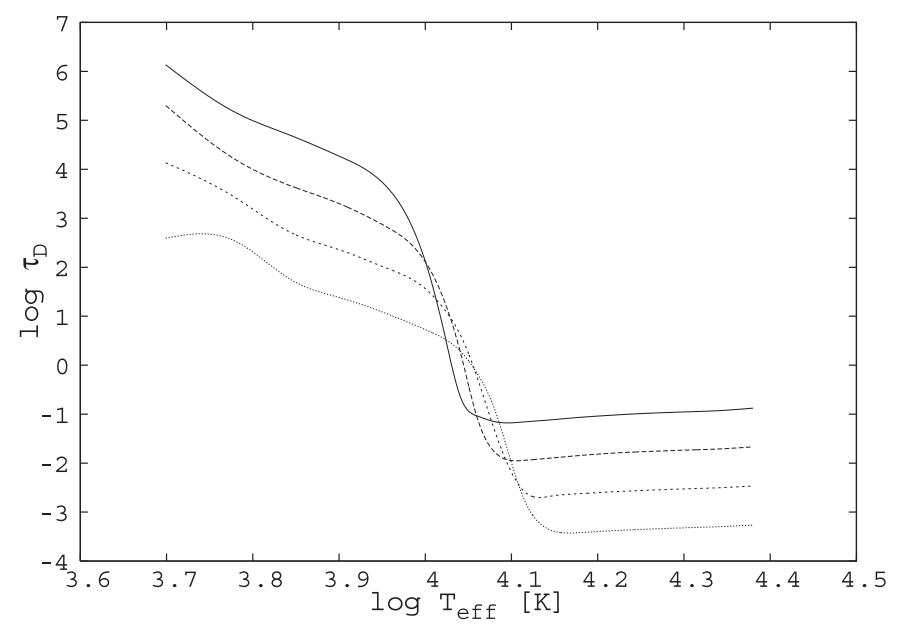

Fig. 2. Diffusion timescales (in years) of Ca. The solid line indicates $\log g=7.5$, then following $\log g=8.0,8.5,9.0$.

decline of a metal abundance means equal time for each decade of abundances and thus a "homogeneous" population of the area above the visibility limit), then the metals will be visible only for a very short time compared to the cooling time through this range - approximately $5.6 \times 10^{8} \mathrm{yr}$ from 20000 to $10000 \mathrm{~K}$, and $1.7 \times 10^{9} \mathrm{yr}$ from 10000 to $6000 \mathrm{~K}$ (Wood 1995). This conflicts with the result that a significant fraction of DA shows metals, $>20 \%$ in Zuckerman et al. (2003) and still $>5 \%$ in Koester et al. (2005), a sample much more biased towards higher $T_{\text {eff }}$. This high fraction is only possible with a constant supply of new DAZs through accretion. On the other hand, taking the numbers for the two-phase scenario at face value, where the star spends on the order of $10^{6} \mathrm{yr}$ in the accretion phase, one would expect a completely different distribution with the vast majority of the objects near the upper boundary of abundances. This leaves little doubt that this interpretation is not correct and that we have to assume that accretion is still ongoing in most, if not all objects. This conclusion is inevitable for the hot objects with timescales of a few days (e.g. about 10 days for G29-38, which has been observed to have Ca since 1997), but is very plausible for all objects. We will thus assume that all DAZ white dwarfs are currently accreting at a steady state (between accretion and diffusion), with this generalization made plausible by the short diffusion timescales (see Dupuis et al. 1992, for a discussion).

\section{Steady state accretion rates}

With the assumption of steady state accretion, we can immediately determine the accretion rate (Dupuis et al. 1993a)

$\dot{M}_{\mathrm{acc}}=\frac{M_{\mathrm{WD}} q X}{\tau_{\mathrm{D}} X_{\mathrm{acc}}}$

with mass fraction of the heavy element in the reservoir $X$ and its abundance in the accreting matter $X_{\text {acc }}$. We assumed the solar abundance of $\mathrm{Ca}$ for $X_{\mathrm{acc}}$ and took the mass fraction $q$ of the reservoir from our models with the appropriate stellar parameters. The resulting mass accretion rates are given in Table 3 and Fig. 3.

The continuous distribution of observed $\mathrm{Ca}$ abundances thus translates into a continuum of derived accretion rates, with a maximum rate of approximately $3 \times 10^{-15} M_{\odot} /$ yr over the whole
Table 3. Diffusion timescales $\tau_{\mathrm{D}}$, mass fraction at the layer of diffusion, $\log q$, and the accretion rates $\dot{M}$ in $M_{\odot} /$ yr for 38 DAZs abundances.

\begin{tabular}{rrrrr}
\hline \hline Object & $\log \tau_{\mathrm{D}}$ & $M_{\mathrm{WD}}$ & $\log q$ & $\log M$ \\
\hline WD0032-175 & 2.72 & 0.600 & -10.04 & -17.84 \\
HS0047+1903 & -1.48 & 0.512 & -15.56 & -14.83 \\
HE0106-3253 & -1.82 & 0.616 & -15.90 & -14.45 \\
WD0208+296 & 3.78 & 0.537 & -8.43 & -15.64 \\
WD0235+064 & -1.04 & 0.550 & -14.91 & -17.69 \\
WD0243-026 & 3.39 & 0.719 & -8.67 & -16.37 \\
WD0245+541 & 4.47 & 0.712 & -6.98 & -17.66 \\
HS0307+0746 & 1.81 & 0.664 & -11.42 & -14.87 \\
WD0408-041 & -1.56 & 0.506 & -15.70 & -15.39 \\
WD0543+579 & 3.22 & 0.597 & -9.17 & -17.48 \\
WD0846+346 & 3.51 & 0.595 & -8.72 & -16.22 \\
WD1015+161 & -1.59 & 0.571 & -15.60 & -14.51 \\
WD1102-183 & 3.27 & 0.597 & -9.10 & -17.25 \\
WD1116+026 & -1.73 & 0.552 & -15.86 & -15.24 \\
WD1124-293 & 2.29 & 0.663 & -10.66 & -15.69 \\
WD1150-153 & -1.63 & 0.500 & -15.79 & -14.82 \\
WD1202-232 & 2.59 & 0.724 & -9.900 & -16.79 \\
WD1204-136 & -0.34 & 0.606 & -14.01 & -15.44 \\
WD1208+576 & 4.50 & 0.530 & -7.31 & -17.25 \\
HE1225+0038 & 2.48 & 0.662 & -10.31 & -17.03 \\
WD1257+278 & 3.26 & 0.541 & -9.25 & -15.24 \\
HE1315-1105 & 2.02 & 0.857 & -10.63 & -16.47 \\
WD1337+705 & -1.57 & 0.574 & -15.56 & -15.29 \\
WD1344+106 & 3.68 & 0.594 & -8.46 & -18.02 \\
WD1407+425 & 2.27 & 0.602 & -10.83 & -17.48 \\
WD1455+298 & 4.33 & 0.386 & -7.98 & -16.38 \\
WD1457-086 & -1.73 & 0.628 & -15.71 & -14.64 \\
WD1614+160 & -1.46 & 0.514 & -15.53 & -15.91 \\
WD1633+433 & 3.69 & 0.654 & -8.34 & -15.17 \\
WD1821-131 & 2.89 & 0.853 & -9.21 & -17.43 \\
WD1826-045 & 2.58 & 0.661 & -10.11 & -15.83 \\
WD1858+393 & 2.58 & 0.601 & -10.30 & -15.27 \\
WD2115-560 & 2.29 & 0.663 & -10.66 & -15.09 \\
HS2132+0941 & -1.47 & 0.454 & -15.63 & -15.97 \\
WD2149+021 & -1.62 & 0.565 & -15.68 & -16.37 \\
HE2221-1630 & 1.84 & 0.727 & -11.28 & -14.82 \\
HS2229+2335 & -1.60 & 0.569 & -15.62 & -14.53 \\
WD2326+049 & -1.70 & 0.551 & -15.81 & -15.13 \\
\hline & & & &
\end{tabular}

range of observed temperatures. The lack of higher abundances below $T_{\text {eff }} \approx 10000 \mathrm{~K}$ in Fig. 1 is due to the bottom of the reservoir moving into deeper and denser regions, as can be seen from the combination of Eqs. (1) and (2):

$X=\frac{\dot{M}_{\mathrm{acc}} X_{\mathrm{acc}} \tau_{\mathrm{D}}}{M_{\mathrm{WD}} q}=\frac{\dot{M}_{\mathrm{acc}} X_{\mathrm{acc}}}{4 \pi r_{\mathrm{b}}^{2} \rho w}$

The radius does not change significantly, but the increased diffusion flux $\rho w$ leads to lower abundances in the observable reservoir. The range of accretion rates is in good agreement with the results of Dupuis et al. (1993b) for helium-envelope white dwarfs; in fact, their "high accretion rate" used for the comparison with observed abundances is $5 \times 10^{-15} M_{\odot} / \mathrm{yr}$, in excellent agreement with Fig. 3. If the maximum accretion rates are indeed independent of the surface temperature of the star, one could even speculate that our mixing-length description predicts convection zone depths at the low temperature end, which are slightly too large! 


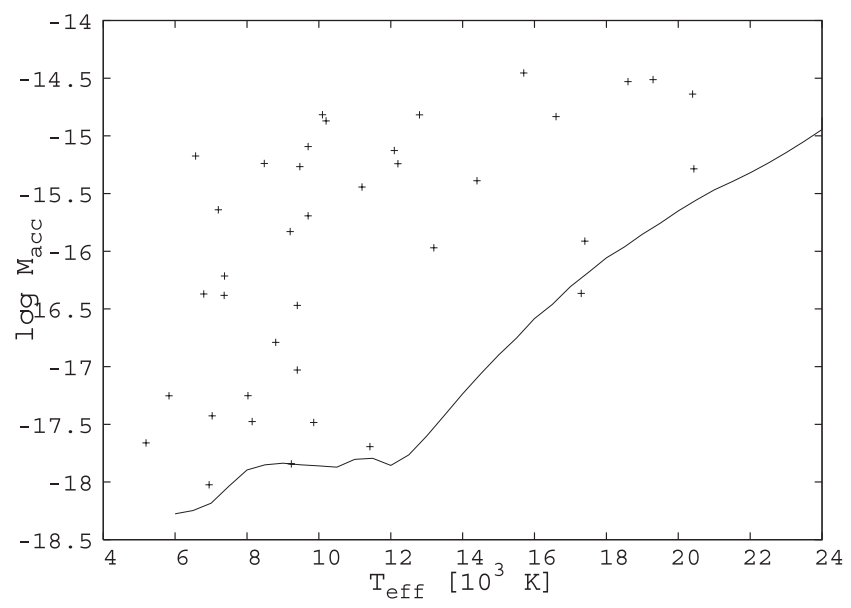

Fig. 3. Accretion rates for $38 \mathrm{DAZ}$ in solar masses per year, assuming that the $\mathrm{Ca}$ abundance in the accreted material is solar. The line shows the limit of the accretion rates resulting from an observational limit of $15 \mathrm{~m} \AA$ for a $0.6 M_{\odot}$ white dwarf.

\section{Correlation of accretion rates with stellar parameters}

What is the reason for the large variation in accretion rates, e.g. by three orders of magnitude at the cool end? To study this question, we have to proceed to the theoretically much less secure ground of the accretion process. Rather than discuss the problems of the physics of accretion, which is beyond the scope of the present study, we assume that the mass accretion rate is given by the Bondi-Hoyle formulation (Hoyle \& Lyttleton 1939; Bondi \& Hoyle 1944; Bondi 1952; Ruffert 1994; Ruffert \& Arnett 1994):

$\dot{M}_{\mathrm{acc}}=\frac{4 \pi G^{2} M^{2} \rho}{\left(c^{2}+v_{\mathrm{s}}^{2}\right)^{3 / 2}}$.

Here, $M$ is the stellar mass, $v_{\mathrm{s}}$ its velocity relative to the interstellar gas at large distance, $c$ the sound velocity and $\rho$ the unperturbed ISM density. For the warm $(\approx 8000 \mathrm{~K})$ phase of the ISM, the sound velocity is not important compared to the space velocity for most objects; we have taken $c=8 \mathrm{~km} \mathrm{~s}^{-1}$ and use a new variable $v=\sqrt{c^{2}+v_{\mathrm{s}}^{2}}$ in the following. The accretion rate therefore can be separated into individual properties of the stars $\left(\propto M^{2} / v^{3}\right)$ and the condition of the ambient medium given by $\rho$. Fortunately, the stellar parameters mass and space velocity (in 8 objects estimated from the radial velocity) are known for our complete sample.

Figure 4 shows accretion rates as a function of the combination $M^{2} / v^{3}$ ("stellar factor"), which enters the Bondi-Hoyle formula Eq. (4). Although the stellar factor varies by more than three orders of magnitude, there is obviously no correlation with the accretion rates. At most one could state that there is a small tendency towards higher rates for large factors and vice versa. This can only mean that the second factor, the interstellar density, must play an equally large role and show similar variations if accretion is coming from the ISM. This is demonstrated in Fig. 5, which shows the distribution of interstellar density (derived from all the other known factors in Eq. (4) and converted to hydrogen particle density) as a histogram. As expected from the lack of correlation in Fig. 3, the derived interstellar densities span the range $n_{\mathrm{H}}=0.01-1 \mathrm{~cm}^{-3}$, with very few objects outside (we use $n_{\mathrm{H}}$ for the sum of neutral and ionized hydrogen atoms). It should be noted that this number is derived from the mass density and thus includes neutral and ionized hydrogen.

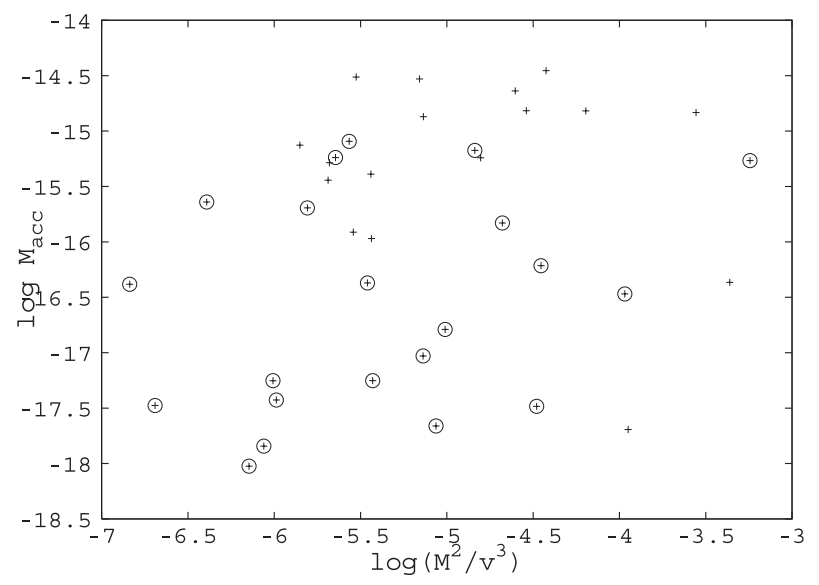

Fig. 4. Accretion rates vs. the "stellar factor" $M^{2} / v^{3}$ for 38 objects. Objects with $T_{\text {eff }}<10000 \mathrm{~K}$ are marked with circles. Accretion rates are in $M_{\odot}$ per year, the units for the stellar factor are $M_{\odot}$ and $\mathrm{km} \mathrm{s}^{-1}$.

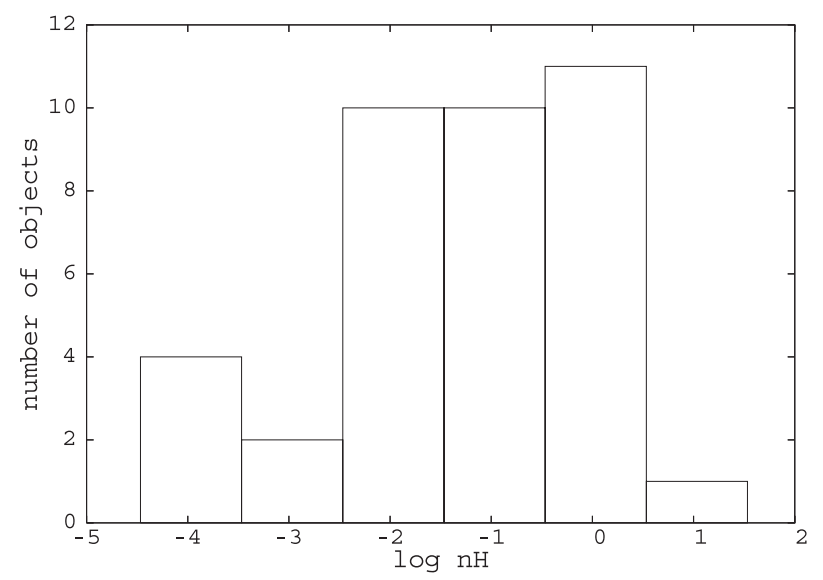

Fig. 5. ISM density (neutral + ionized hydrogen per $\mathrm{cm}^{3}$ ) derived from assuming the validity of the Bondi-Hoyle accretion formula. See text.

The direct (not $\log$ ) average of all values is $\approx 0.5$, which is very likely biased towards higher values, because for higher $T_{\text {eff }}$ we can observe only the highest accretion rates.

For most of the current sample the distances are known, and we can therefore determine the location in the solar neighborhood corresponding to the individual density determinations. We can also derive upper limits for the ISM densities for many of the numerous objects with upper limits for the $\mathrm{Ca}$ abundance (Fig. 1). We had hoped to find examples of several DAZs close together in an area of the sky, all with enhanced ISM densities derived from the accretion rates, but no such correlations are apparent. If our interpretation up to this point is at all meaningful, this means that the ISM density varies by two orders of magnitude on the scale of a few pc.

\section{Discussion and conclusions}

The two-phase accretion scenario in the highly idealized form of the Dupuis et al. work cannot explain the observations of the DAZs discussed in this study. The absence of cold neutral matter in the solar neighborhood, the large fraction of DA showing metals in spite of short diffusion timescales, and the distribution of the observed abundances all point to ongoing accretion even under the conditions of the ISM in the Local Bubble.

Although we show that the stellar parameters entering the calculation of accretion rates according to Bondi-Hoyle 
hydrodynamic accretion vary over several orders of magnitude, this alone cannot explain the variation in observed accretion rates. Additionally, we need a significant variation in the ISM densities, with typical values of ionized plus neutral hydrogen $n_{\mathrm{H}} \approx 0.1-0.2 \mathrm{~cm}^{-3}$ and variation by a factor of 10 in both directions. The only viable candidate is the warm, partially ionized phase of the solar neighborhood ISM, which has been studied recently in a series of papers by Linsky et al. (2000) and Redfield \& Linsky (2000, 2002, 2004a,b).

From these papers the following picture emerges. The absorption lines along the line-of-sight to many nearby stars show the existence of distinct components, tentatively called "clouds". Neutral hydrogen densities are typically $n_{\mathrm{HI}} \approx 0.1 \mathrm{~cm}^{-3}$, with hydrogen ions adding $n_{\mathrm{p}} \approx 0.11 \mathrm{~cm}^{-3}$ to the mass. The length scale is $2.2 \mathrm{pc}$, with a range of 0.1 to $11 \mathrm{pc}$. Our own sun lies within a similar cloud, the Local Interstellar Cloud or LIC, with extensions of 4-6 pc. We use these numbers for some very simple estimates, to test the plausibility of a connection between these clouds and the DAZ phenomenon.

Assuming current accretion within such a cloud to be responsible for the observed metals, the observations demand a filling factor on the order of $10 \%$ within $50 \mathrm{pc}$. Taking spherical clouds with a radius of $1.5 \mathrm{pc}$, the total number of clouds in this volume would be 3700 . The total solid angle covered by these clouds (distributed homogeneously) is 31.4 or 2.5 times the total sphere of $4 \pi$. This is in excellent agreement with the findings of $1-3$ components (and an average of $\approx 2$ ) along each line-of-sight in the Redfield and Linsky studies. A white dwarf with a space velocity of $30 \mathrm{~km} \mathrm{~s}^{-1}$ would travel $1 \mathrm{pc}$ in about $33000 \mathrm{yr}$, plenty of time to reach steady state abundances and stay constant over the observational timescales.

The typical distance between the centers of any two clouds would be $3.2 \mathrm{pc}$, about the same as the size of the clouds. This should serve as a caveat and a reminder that the concept of "clouds" may again be an oversimplification. However, this does not affect our interpretation, since all we need is a scale length for significant variation in the physical parameters of the ISM. These estimates are all quite favorable to the hypothesis of ongoing accretion from the warm phase of the ISM, alleviating the strongest objection against the accretion/diffusion scenario - the absence of cool, neutral clouds.

There are, however, other open questions remaining. One of them is whether the assumption of fluid (Bondi-Hoyle) accretion rates is justified. Koester (1976) concluded that accretion rates would be much lower than the fluid rate since interactions are not sufficient to destroy the momentum perpendicular to the accretion column, a necessary condition for the hydrodynamic treatment. On the other hand, Alcock \& Illarionov (1980) argued that an ionized plasma would always accrete at the fluid rate due to plasma instabilities and magnetic fields.

Another difficult question is the composition of the accreted matter. We have derived a very robust result on the accretion of $\mathrm{Ca}$ atoms (which can be derived from the mass accretion rates in Table 3 by multiplying those numbers by $8 \times 10^{-5}$ ). The total accretion rate follows from assuming a solar composition. There is no evidence for that; on the contrary, observations in helium-rich white dwarfs with metals indicate that in many cases very little or no hydrogen is accreted. This seems to show that the accretion is preferentially of dust grains, either interstellar or from some circumstellar cloud or disk. Circumstellar material has been found for G29-38 and GD362 (see e.g. Jura 2003; Reach et al. 2005; Becklin et al. 2005; Kilic et al. 2005, for recent studies), with the origin attributed to comets, tidal disruption of planets or asteroids, or the merging of two white dwarfs
(Livio et al. 2005). It is quite possible that in such cases accretion from a different source than the ISM is responsible for the observed metals; similarly, in several cases of close M dwarf companions (Zuckerman et al. 2003), a stellar wind may provide the heavy elements.

However, these seem to be exceptional cases. As found in the comprehensive recent work by Farihi et al. (2005) none of the 371 white dwarfs studied showed a near-infrared excess indicative of circumstellar dust similar to G29-38 and GD362. One should notice that the search was limited by the signalto-noise ratio of the Ks magnitude in the 2MASS sample and only about $1 / 3$ of the sample had adequate data, so further detections may be forthcoming from ongoing searches (J. Farihi, priv. comm.). Nevertheless, it appears unlikely that such processes can account for the 10-20\% of hydrogen-rich white dwarfs that are below $20000 \mathrm{~K}$ and have heavy elements.

Acknowledgements. We are grateful to Ben Zuckerman and Michael Jura for stimulating discussions. This work was supported in part by a grant from the Deutsche Forschungsgemeinschaft (Ko738/21-1,2).

\section{References}

Aannestad, P. A., Kenyon, S. J., Hammond, G. L., \& Sion, E. M. 1993, AJ, 105, 1033

Alcock, C., \& Illarionov, A. 1980, ApJ, 235, 541

Becklin, E. E., Farihi, J., Jura, M., et al. 2005, ApJ, 632, L119

Berger, L., Koester, D., Napiwotzki, R., Reid, I. N., \& Zuckerman, B. 2005, A\&A, 444, 565

Bondi, H. 1952, MNRAS, 112, 195

Bondi, H., \& Hoyle, F. 1944, MNRAS, 104, 273

Chayer, P., Vennes, S., Pradhan, A. K., et al. 1995, ApJ, 454, 429

Dupuis, J., Fontaine, G., Pelletier, C., \& Wesemael, F. 1992, ApJS, 82, 505

Dupuis, J., Fontaine, G., Pelletier, C., \& Wesemael, F. 1993a, ApJS, 84, 73

Dupuis, J., Fontaine, G., \& Wesemael, F. 1993b, ApJS, 87, 345

Farihi, J., Becklin, E. E., \& Zuckerman, B. 2005, ApJS, 161, 394

Fontaine, G., Villeneuve, B., \& Wilson, J. 1981, ApJ, 243, 550

Hoyle, F., \& Lyttleton, R. A. 1939, in Proceedings of the Cambridge Philisophical Society, 405

Jura, M. 2003, ApJ, 584, L91

Kilic, M., von Hippel, T., Leggett, S. K., \& Winget, D. E. 2005, ApJ, 632, L115

Koester, D. 1976, Ph.D. Thesis, Universität Kiel, Germany

Koester, D., Rollenhagen, K., Napiwotzki, R., et al. 2005, A\&A, 432, 1025

Lehner, N., Jenkins, E. B., Gry, C., et al. 2003, ApJ, 595, 858

Linsky, J. L., Redfield, S., Wood, B. E., \& Piskunov, N. 2000, ApJ, 528, 756

Livio, M., Pringle, J. E., \& Wood, K. 2005, ApJ, 632, L37

McCook, G. P., \& Sion, E. M. 1999, ApJS, 121, 1

Muchmore, D. 1979, in White Dwarfs and Variable Degenerate Stars, IAU Coll., 53, 197

Muchmore, D. 1984, ApJ, 278, 769

Paquette, C., Pelletier, C., Fontaine, G., \& Michaud, G. 1986a, ApJS, 61, 177 Paquette, C., Pelletier, C., Fontaine, G., \& Michaud, G. 1986b, ApJS, 61, 197 Pauli, E. 2003, Ph.D. Thesis, Universität Erlangen-Nürnberg

Reach, W. T., Kuchner, M. J., von Hippel, T., et al. 2005, ApJ, 635, L161

Redfield, S., \& Linsky, J. L. 2000, ApJ, 534, 825

Redfield, S., \& Linsky, J. L. 2002, ApJS, 139, 439

Redfield, S., \& Linsky, J. L. 2004a, ApJ, 602, 776

Redfield, S., \& Linsky, J. L. 2004b, ApJ, 613, 1004

Ruffert, M. 1994, ApJ, 427, 342

Ruffert, M., \& Arnett, D. 1994, ApJ, 427, 351

Schatzman, E. 1947, C.R., 225, 990

Sfeir, D. M., Lallement, R., Crifo, F., \& Welsh, B. Y. 1999, A\&A, 346, 785

Tassoul, M., Fontaine, G., \& Winget, D. E. 1990, ApJS, 72, 335

Welsh, B. Y., Crifo, F., \& Lallement, R. 1998, A\&A, 333, 101

Welsh, B. Y., Sfeir, D. M., Sirk, M. M., \& Lallement, R. 1999, A\&A, 352, 308

Wood, M. A. 1995, in White Dwarfs, Proceedings of the 9th European Workshop on White Dwarfs Held at Kiel, Germany, 29 August-1 September 1994, XII, 348 pp. (Berlin, Heidelberg, New York: Springer-Verlag), Also Lecture Notes in Physics, Vol. 443, ed. D. Koester, \& K. Werner, 51

Zuckerman, B., Koester, D., Reid, I. N., \& Hünsch, M. 2003, ApJ, 596, 477 Zuckerman, B., \& Reid, I. N. 1998, ApJ, 505, L143 